\title{
Le droit du travail au-delà des frontières nationales : Principaux débats en 2017
}

\section{Mariapaola Aimo, Rudolf Buschmann et Daniela Izzi}

\section{(2) OpenEdition}

1 Journals

\section{Édition électronique}

URL : https://journals.openedition.org/rdctss/1569

DOI : 10.4000/rdctss. 1569

ISSN : 2262-9815

Éditeur

Centre de droit comparé du travail et de la sécurité sociale

\section{Édition imprimée}

Date de publication : 1 juin 2019

Pagination : 124-147

ISSN : 2117-4350

\section{Référence électronique}

Mariapaola Aimo, Rudolf Buschmann et Daniela Izzi, « Le droit du travail au-delà des frontières nationales : Principaux débats en 2017 », Revue de droit comparé du travail et de la sécurité sociale [En ligne], 2 | 2019, mis en ligne le 01 novembre 2021, consulté le 11 novembre 2021. URL : http:// journals.openedition.org/rdctss/1569; DOI : https://doi.org/10.4000/rdctss.1569

\section{(c) $(1) \&$}

Revue de droit comparé du travail et de la sécurité sociale est mise à disposition selon les termes de la Licence Creative Commons Attribution - Pas d'Utilisation Commerciale - Pas de Modification 4.0 International. 


\section{LitTÉRATURE DE DROIT SOCIAL COMPARÉ}

MARIAPAOLA AIMO

Professeur associé, Université de Turin, Département de droit

\section{RUDOLF BUSCHMANN}

Maître de conférences associé à l'Université de Kassel ancien Rédacteur en Chef Arbeit und Recht

\section{DANIELA IZZI}

Professeur associé, Université de Turin, Département de droit

\section{LE DROIT DU TRAVAIL AU-DELÀ DES FRONTIÈRES NATIONALES : PRINCIPAUX DÉBATS EN 20171}

Le présent article donne un aperçu des principaux sujets abordés dans 24 des 30 revues membres de l'Association internationale des revues de droit du travail (IALLJ) au cours de l'année 2017. Les autres revues n'ont pas été étudiées, en raison principalement de leur difficulté d'accès ou de la barrière linguistique ${ }^{3}$. Cette analyse est la sixième du genre depuis 2013, date à laquelle la première " édition » a été publiée par Lavoro e diritto et la Revue de Droit comparé du Travail et de la Sécurité sociale. Cette synthèse se divise en trois parties : I) Vers une société du travail inclusive ?; II) Transformations du travail : les nouveaux défis et les nouveaux risques « mettent à l'épreuve » la législation; III) Perspectives pour le droit du travail collectif.

\section{I - VERS UNE SOCIÉTÉ DU TRAVAIL INCLUSIVE ?}

De nombreux articles publiés dans les revues examinées portent sur les efforts qui doivent être entrepris pour construire des sociétés plus inclusives ; un objectif ambitieux

1 Bien que cette étude soit le résultat d'un travail en commun des trois auteurs, le $\S 1$ a été rédigé par D. Izzi, le $\S 2$ par M. Aimo et le $\S 3$ par R. Buschmann. Les auteurs tiennent à remercier leurs collègues (Gian Guido Balandi, Marialaura Birgillito, Silvia Borelli, Matteo Borzaga, Isabelle Daugareilh, Sebastián de Soto Rioja, Manuel Antonio García-Muñoz Alhambra, Eva Maria Hohnerlein, Eri Kasagi, Barbara Kresal, Sandrine Laviolette, Steven Willborn) qui les ont aidés à lister et traduire les index des revues prises en compte. Le présent document est dédié à la mémoire de notre collègue Sandrine Laviolette.

2 Pour accéder à la liste complète, consulter www.labourlawjournals.com. La liste de toutes les abréviations des revues mentionnées dans cet article figure à la fin.

3 Nous nous référons aux revues suivantes : Análisis Laboral (Pérou), Industrial Law Journal (Afrique du Sud), Labour and Social Law (Biélorussie), Labour Society and Law (Israël), Pecs Labour Law Journal (Hongrie) et Russian Yearbook of Labour Law (Russie). 
au service duquel le droit du travail et les systèmes de sécurité sociale aux niveaux national et international ont été particulièrement mobilisés.

À cet égard, les règles anti-discrimination qui visent à surmonter ce problème largement connu dans le monde du travail - du traitement défavorable des personnes appartenant à des catégories dites «à risque», jouent incontestablement un rôle important, et ce partout dans le monde. En effet, de nombreux articles de I'IALLJ parus en 2017 traitent des dispositions spécifiques du droit anti-discrimination et examinent l'impact potentiellement négatif du sexe, de l'âge, du handicap, de la race ou de la religion ( $\mathbf{A}$, B et D) sur l'emploi. D'autres articles, pour leur part, abordent la conciliation entre la vie professionnelle et la vie familiale dans les sociétés du travail en l'examinant sous l'angle du sexe, de l'âge et du handicap (C).

Une attention particulière a également été accordée au débat plus général portant sur les obstacles actuels à la lutte juridique contre les différents types de discrimination, qui freinent l'émergence d'une véritable égalité dans le monde du travail. Comme l'a montré le dialogue mené sur ce sujet entre spécialistes de différents pays de Common Law, il existe des lacunes procédurales ou institutionnelles, même dans des pays ayant une longue expérience en matière de protection contre les discriminations à l'instar des États-Unis et du Royaume-Uni. A cet égard, des difficultés encore plus importantes sont apparues en Australie, en dépit de la réforme juridique adoptée il y a quelques années qui a permis aux salariés de déposer plainte pour discrimination en dehors du seul cas de licenciement ${ }^{4}$.

Du point de vue européen, "l'effet transformateur » de I'UE sur la législation antidiscrimination britannique est particulièrement intéressant dans ce débat international. Cet effet est dû en grande partie à « l'approche intentionnelle et centrée sur les droits » adoptée par la Cour de justice, dont l'influence peut perdurer par-delà le Brexit 5 . Sur le vieux continent, le débat sur la construction de sociétés de travail inclusives a également

4 A. Chapman, B. Gaze, A. Orifici, "Substantive equality at work: Still elusive under Australia's Fair Work Act ", AJLL, 2017, vol. 30, p. 214. Ces auteurs ont décrit les résultats décevants de la réforme de 2009 de la loi sur le travail équitable (Fair Work Act) en Australie, qui a été perçue comme une occasion de faciliter les recours contre les discriminations au travail et de réduire les traitements illégaux défavorables. Concernant le problème spécifique de l'approche subjective qui n'a pas été complètement abandonnée dans la gestion judiciaire des plaintes des salariés contre les discriminations et les actions défavorables, se référer à « Australian courts approaches to unconscious direct discrimination and adverse action ", AJLL, 2017, vol. 30, p. 1, et à E. Shi, "Adverse action protection for the right to complain or inquire in s 341 of the Fair Work Act ", AJLL, 2017, vol. 30, p. 294.

5 O'Cinneide, "Values, rights and Brexit - Lessons to be learnt from the slow evolution of United Kingdom discrimination law», AJLL, 2017, vol.30, p. 236. Toutefois, comme l'a souligné O'Cinneide, la CEDH a également exercé une influence positive sur l'évolution du droit britannique en matière d'égalité. Plus généralement, au sujet de la pertinence pour le droit du travail de l'interdiction de la discrimination établie par l'article 14 de la Convention, voir E. Sychenko, "Individual Labour Rights as Human Rights. The Contributions of the European Court of Human Rights to Worker's Rights Protection », BCLR, 2017, vol. 96, p. 67. 
soulevé de nombreuses questions liées aux incertitudes qui frappent le modèle social européen à l'heure du Brexit' .

\section{A - LA QUÊTE PERMANENTE DE L'ÉGALITÉ DES SEXES}

C'est sans surprise que la question des inégalités entre les sexes - problème ancien mais toujours non résolu - est restée à l'ordre du jour dans les revues de l'IALLJ parues en 2017. Eu égard au caractère très large de la notion de l'égalité salariale, ce sujet a fait l'objet de réceptions diverses selon la région du monde considérée. Les différents systèmes juridiques retiennent en effet des approches réglementaires qui présentent de nombreuses disparités ${ }^{7}$, au regard notamment des critères utilisés pour déterminer si l'objectif de la parité salariale pour un même emploi est atteint (ici, le choix du comparateur est un élément $\mathrm{crucia}^{8}{ }^{8}$, si les plaintes individuelles ou collectives sont autorisées, et pour définir la portée des recours offerts aux demandeurs?

En revanche, les questions relatives aux droits liés à la maternité, à la protection contre la discrimination des femmes qui travaillent pendant leur grossesse, à la période postpartum et à l'allaitement, ont davantage été soulevées en Europe ${ }^{10}$. Ces discussions (que l'on retrouve principalement dans les revues espagnoles, mais aussi en Allemagne où une nouvelle loi sur la protection de la maternité est sur le point d'entrer en vigueur) ont également permis de mettre en exergue les nouvelles questions juridiques posées par la fécondation in vitro ${ }^{11}$ et la gestation pour autrui ${ }^{12}$.

Un article intéressant relatif à la prévention des risques professionnels des femmes en général, en dehors du contexte de la maternité, souligne l'urgence de dépasser les normes androcentriques traditionnelles adoptées par la réglementation sur la santé et

6 Voir E. Ales, «Il Modello Sociale Europeo dopo la crisi: una mutazione genetica ?», DLM, 2017, 3, p. 485; J. Kenner, « II potenziale impatto della Brexit sul Diritto del lavoro europeo e britannico ", $D L M, 2017,1$, p. 5; M. Weiss, "The future of labour law in Europe. Rise or fall of the European social model ?», ELLJ, 2017, 4, p. 345. Avec un accent particulier sur le pilier social européen, S. Laulom, J-P. Lhernould, "Quelle Europe sociale nous prépare le socle des droits sociaux? », Revue de Droit du Travail, 2017, 7-8, p. 455 ; K. Lörcher, « Die Europäische Säule Sozialer Rechte - Rechtsfortschritt oder Alibi ?», 2017, 10, p. 387.

7 M. Smith, R. Layton, A. Stewart, «Inclusion, Reversal, or Displacement: Classifying Regulatory Approaches to Pay Equity », CLLPJ, 2017, vol. 39, p. 211.

8 M. Smith, A. Stewart, "Shall I compare thee to a fitter and turner? The role of comparators in pay equity regulation ", AJLL, 2017, vol. 30, p. 113.

9 Dans un autre ordre d'idées, le potentiel des accords internationaux de libre-échange dans l'amélioration des conditions de travail des femmes qui travaillent dans les chaînes d'approvisionnement mondiales est exposé par T.J. Brooks, « Undefined Rights : The Challenge of Using Evolving Labor Standards in U.S. and Canadian Free Trade Agreements to Improve Working Women's Lives », CLLPJ, 2017, vol. 39, p. 29.

10 K. Nebe, B. Graue, "Die Reform des Mutterschutzgesetzes », AuR, 2017, 11, p. 437 ; S. Rodríguez et S. Escanciano, "La regulación de la lactancia en el ordenamiento laboral: algunas cuestiones pendientes », DRL, 2017, 5, p. 402 ; J.M. Serrano García, « La situación de discriminación directa de la mujer en situación de maternidad por incumplimiento de clausola convencionales de reconocimiento de derechos », RDS, 2017, 78, p. 149 ; M.V. Ballestrero, " Anna Kuliscioff, il lavoro e la cittadinanza delle donne. Uno sguardo dal presente $», L D, 2017,2$, p. 187.

11 M.B. Cardona Rubert, « Discriminación por fecundación in vitro: la nueva frontera al derecho de la trabajadora a ser madre $», R D S, 2017,79$, p. 185.

12 B.d.M.López Insua, "Maternidad subrogada y protección del menor desde una perspectiva integradora: el derecho laboral de nuevo a examen », DRL, 2017, 2, p. 166. 
la sécurité des travailleurs, arguant des différences significatives entre les sexes relevées dans les études biomédicales ${ }^{13}$. Cette révision pourrait être considérée comme un exemple marquant d'application de la méthode féministe aux questions de droit du travail, qu'il serait réducteur d'interpréter comme une simple conséquence des préoccupations d'égalité entre les sexes, omettant ainsi son utilité conceptuelle plus large pour l'organisation sociale du travail et sa réglementation ${ }^{14}$.

\section{B - LES PERSONNES ÂGÉES ET HANDICAPÉES SUR LE MARCHÉ DU TRAVAIL}

Les exigences spécifiques que pose le vieillissement de la population pour le droit du travail et les systèmes de sécurité sociale ont été au centre de plusieurs articles publiés dans les revues de l'IALLJ, en Europe particulièrement mais pas exclusivement ${ }^{15}$.

Les auteurs se sont concentrés sur les différentes mesures qui peuvent s'avérer utiles pour prolonger les carrières professionnelles et améliorer la place des personnes âgées dans la vie active, même après l'âge de la retraite. Parmi ces outils, on peut citer l'interdiction de la discrimination fondée sur l'âge et le renforcement des mesures juridiques pour la prévenir ${ }^{16}$, ainsi que des dispositifs incitatifs tels que des programmes de formation offerts par les employeurs ${ }^{17}$. L'emploi des personnes âgées, contrairement à la théorie de la " masse de travail fixe» (lump of labour) qui a encouragé l'expansion des politiques de retraite anticipée au cours des dernières décennies, contribue à créer un lien favorable avec le travail des jeunes ${ }^{18}$. II n'y a dès lors aucune raison scientifique de s'opposer à la protection face au marché du travail des personnes âgées comme des jeunes ${ }^{19}$.

Les changements démographiques d'une part, et l'impulsion donnée par la Convention des Nations Unies relative aux droits des personnes handicapées (ratifiée par I'UE en 2010) d'autre part, expliquent sans doute l'intérêt généralisé des spécialistes de l'IALLJ pour le handicap et les dispositions anti-discrimination connexes.

L'évolution de la notion de handicap, telle que préconisée par la Convention et mise en application dans la jurisprudence récente de la CJUE concernant des situations d'incapacité de travail temporaire et de maladie de longue durée, a exercé une forte

13 A. Garrigues Giménez, « Hacia un nuevo paradigma (no androcéntrico) en la prevención de riesgos laboral es la necesaria e inaplazable integración normativa y técnica del diferencial de sexo y de género », DRL, 2017, 8, p. 763.

14 J. Conaghan, "Labour Law and Feminist Method », IJCLLIR, 2017, 1, p.93.

15 Sur la situation au Japon, voir K. Inamori, « Current Situation and Problems of Legislation on LongTerm Care in Japan's Super-Aging Society », JLR, 2017, vol. 14, 1, p. 8.

16 A. Vaitkeviciute, «Prohibition of Age Discrimination in the Labour Market - Case Study of Finland in the Context of European Union », E\&E, 2017, 1, p. 9. Cet auteur examine le modèle réglementaire avancé de la Finlande.

17 P.B. Berg, M.K. Hamman, M.M. Piszczek, C.J. Ruhm, «The relationship between employer-provided training and the retention of older workers: Evidence from Germany ", ILR, 2017, 3-4, p. 495. Et, pour des conclusions quelque peu différentes, voir F.G. Gommans, N.W.H. Jansen, D. Stynen, I. Kant, A. De Grip, «The effects of under-skilling on need for recovery, losing employment and retirement intentions among older office workers: A prospective cohort study ", ILR, 2017, 3-4, p. 525.

18 Pour une analyse approfondie, voir E. Jeong, «The relationship between youth employment and older persons' employment in 20 OECD countries », ILR, 2017, 3-4, p. 425.

19 Sur ce dernier point, voir M. Rodríguez-Piñero y Bravo-Ferrer, « Discriminación por razón de edad y trabajadores jóvenes », DRL, 2017, 11, p. 1033. 
pression sur les règlementations traditionnelles en matière de travail, notamment s'agissant des licenciements ${ }^{20}$. Cette pression résulte en partie de l'obligation pour l'employeur de fournir des installations adaptées aux travailleurs handicapés. Cette prescription joue un rôle crucial pour la pleine intégration et l'égalité des personnes handicapées, et fait l'objet d'une analyse portant sur le degré d'aménagement du lieu de travail auquel les employeurs sont aujourd'hui assujettis ${ }^{21}$.

Il est évident que les efforts juridiques visant à promouvoir l'intégration des personnes handicapées sur le marché du travail peuvent contribuer à réduire de manière significative les dépenses publiques consacrées aux pensions d'invaliditée ${ }^{2}$. Même si les politiques classiques du marché du travail demeurent la réponse privilégiée à la nécessité d'accompagner les personnes handicapées au retour à l'emploi, un certain nombre de politiques innovantes de "gestion inclusive des ressources humaines » ont tenté, ces dernières années, d'impliquer les employeurs dans cette tâche difficile ${ }^{23}$.

\section{C - LE BESOIN GÉNÉRALISÉ D’UN ÉOUILIBRE ENTRE VIE PROFESSIONNELLE ET VIE PRIVÉE}

Un débat animé, auquel ont participé des chercheurs du monde entier, a porté sur le thème très complexe de la conciliation entre vie professionnelle et vie privée. Ici, la vie privée désigne principalement la vie familiale et implique donc le rôle des travailleurs en tant que soignants de proches dépendants : les enfants principalement ${ }^{24}$, mais aussi et de plus en plus en raison de l'évolution démographique, les personnes âgées ${ }^{25}$.

Les auteurs qui ont contribué à ce débat ont présenté un très large éventail de propositions, partant du principe que si l'équilibre entre vie professionnelle et vie privée relève en grande partie de la responsabilité individuelle, il nécessite aussi le soutien des

20 L.J. Dueñas Herrero, «La situación de incapacidad temporal puede ser motivo de discriminación? La fuerza del concepto evolutivo de discapacidad integrado en la Directiva 2000/78/CE », DRL, 2017, 5, p. 425; S. Fernández Martínez, «L'evoluzione del concetto giuridico di disabilità: verso l'inclusione delle malattie croniche? ", DRI, 2017, 1, p. 74; J. Moreno Gené, «El dificil recurso a la 'enfermedad asimilada a la discapacidad' como limite del despido por absentismo del trabajador », RDS, 2017, 80, p. 163.

21 E. Purdue, "Scoping reasonable adjustments in the workplace: A comparative analysis of an employer's obligation to accommodate a worker's disability under Australian and Canadian laws », AJLL, 2017, vol. 30, p. 185; J. Harmgardt, «Survival of the Fittest: The Failure to Accommodate and Compensate in the Canadian Armed Forces », CL\&ELJ, 2017, vol. 20, p. 379; C. Spinelli, " La sfida degli 'accomodamenti ragionevoli' per i lavoratori disabili dopo il Jobs Act », DLM, 2017, 1, p. 44.

22 N. Jakab, I. Hoffman, G. Konczei, « Rehabilitation of people with disabilities in Hungary - Questions and Results in Labour Law and Social Law», ZIAS, 2017, vol. 31, 1, p. 23; J. Gorelli Hernández, «El problemático control de la incapacidad temporal en el régimen general»,TL, 2017, vol. 136, p. 13.

23 I. Borghouts-van de Pas, C. Freese, «Inclusive HRM and Employment Security for Disabled People: An Interdisciplinary Approach », E-JICLS, 2017, vol. 6, 1.

24 Voir, inter alia, S. Bernstein, «Addressing Work-Family Conflict in Quebec: The Gap between Policy Discourse and Legal Response », CL\&ELJ, 2017, vol. 20, p. 273; B. García Romero, "La conciliation des responsabilités professionnelles et familiales en cas de maladie grave des enfants à charge en Espagne », Revue de Droit Comparé du Travail et de la Sécurité Sociale, 2017, 1, p. 82.

25 Comme le soulignent K. Inamori, op.cit., et M. Kurzynoga, « Working and Caring - Polish Regulations in the Context of Demographic Changes », E-JICLS, 2017, vol. 6, 1. 
politiques à différents niveaux ${ }^{26}$. En raison de la grande variété des questions abordées dans les articles - qu'il s'agisse de sujets traditionnels relatifs à l'aménagement du temps de travail et aux congés parentaux ${ }^{27}$, à des corrélations plus inhabituelles établies entre le déséquilibre travail-famille et les emplois courts, irréguliers ou mal payés ${ }^{28}$ - il est difficile de résumer succinctement leur contenu ou leurs conclusions. II convient toutefois de mentionner quelques éléments de réflexion convergents, tels le fait de suggérer que les entreprises peuvent jouer un rôle important en encourageant les soignants salariés dans leur " gestion des services de soins et la division des tâches ${ }^{29}$, ou encore la nécessité de remédier au faible taux de participation aux congés pour soins prévus par la législation au Japon et au risque corrélatif de démission qui y est constaté ${ }^{30}$.

Ces éléments illustrent l'impératif de dépasser le concept du travailleur idéal comme étant celui qui n'a pas de responsabilitésfamiliales; une représentation qui prévautcependant toujours dans la législation et qui se trouve renforcée au quotidien par les organisations ${ }^{31}$. La création d'un modèle normatif plus réaliste et plus durable serait certainement utile aux femmes et aux mères qui travaillent, et dont les obligations parentales limitent encore trop leurs chances sur le marché du travail ou dans leur carrière professionnelle ${ }^{32}$. Plus subtilement, cette révision conceptuelle profiterait aussi considérablement aux hommes et aux pères, en récusant le postulat de l'homme soutien de famille selon lequel toute

26 Plus précisément, l'intention de « combler le fossé entre la recherche et la politique en rassemblant les résultats académiques de plusieurs disciplines » a inspiré le volume édité par S. De Groof, "Work-Life Balance in the Modern Workplace. Interdisciplinary Perspectives from Work-Family Research, Law and Policy », BCLR, 2017, vol. 98.

27 M. Basterra Hernández, «Las reducciones y adaptaciones de jornada en atención a las necesidades personales y familiares del trabajador»,RDS, 2017, 78, p. 97 ; S. De Groof, " How Can Labour Law Contribute to Work-Life Balance? Recommendations for a Modern Working Time Law ", BCLR, 2017, vol. 98, p. 51; N. Selberg, "Regulating Work-Life Balance: The Contemporary Swedish Experience », BCLR, 2017, vol. 98, p. 311; G. Vermeylen, A. Parent-Thirion, M. Wilkens, J. Cabrita, "Reconciliation of Work and Private Life as Key Element for Sustainable Work Throughout the Life Course », BCLR, 2017,vol. 98, p. 359.

28 T. Warren, «Work-Life Balance, Time and Money: Identifying the Work-Life Balance Priorities of Working Class Workers », BCLR, 2017, vol. 98, p. 311; A. Masselot, " Precarious Work and WorkFamily Reconciliation: A Critical Evaluation of New Zealand's Regulatory Framework », BCLR, 2017, vol. 98, p. 285.

29 Y. Yajima, «Frameworks for Balancing Work and Long-Term Care Duties, and Support Needed from Enterprises », JLR, 2017, vol. 14, 1, p. 68.

30 Voir respectivement K. Inamori, op.cit., et S. Ikeda, «Family Care Leave and Job Quitting due to Caregiving: Focus on the Need for Long-Term Leave », JLR, 2017, vol. 14, 1, p. 25.

31 Voir S. Bernstein, op.cit., I. Matzner-Heruti, « This Is Not an Ideal, Man: Restructuring the Ideal Worker Norm », BCLR, 2017, vol. 98, p. 183.

32 Comme l'a fait remarquer B. Kresal, «La conciliation travail-famille et l'égalité professionnelle entre les femmes et les hommes en Slovénie », Revue de Droit Comparé du Travail et de la Sécurité Sociale, 2017, 1, p. 58. Plus particulièrement, à propos des conséquences négatives liées à la décision de devenir mère, voir S. Turki, «Work-Family Balance: Origins, Practices and Statistical Portrait from Canada and France », E-JICLS, 2017, vol. 6 ; G. Özcüre, N. Eryigit, H. Demirkaya, "Work-Life Balance in the Modern Workplace: A Comparative Analysis of the Turkish and European Multinational Companies Operating in Turkey », BCLR, 2017, vol. 98, p. 231. 
dispersion dans les rôles traditionnellement masculins compromettrait aussi bien son statut professionnel que sa virilité ${ }^{33}$.

\section{D - SOCIÉTÉS MULTIETHNIQUES ET TRAVAIL: LA QUESTION DU VOILE ISLAMIQUE ET LES DÉFIS DE L'IMMIGRATION}

II n'est pas surprenant qu'en 2017, comme les années précédentes, de nombreux articles de I'IALLJ aient concerné les multiples implications juridiques inhérentes à l'augmentation continue des migrations de main-d'œuvre dans le monde, avec les défis qui en résultent. Cependant, un nouveau sujet lié à nos sociétés multiethniques a commencé à retenir l'attention en 2017, à savoir le conflit - déclenché par le port du voile islamique au travail - entre la liberté de religion des salariés et l'intérêt des employeurs à poursuivre une politique de neutralité.

Bien que la question de la protection contre la discrimination religieuse soit également débattue hors de l'Europe (par exemple au Canada - à nouveau sur la tenue des musulmanes -, où le modèle du multiculturalisme adopté pour gérer la diversité est critiqué par les spécialistes pour des raisons opposées ${ }^{34}$ ), c'est sur le vieux continent que la discussion est la plus animée et ce n'est pas un hasard. Dans les arrêts Achbita et Bougnaoui - deux affaires impliquant le licenciement de musulmanes pour avoir refusé d'ôter leur foulard face à des clients - la CJUE a dû, pour la première fois, se pencher sur le problème sensible des limites aux droits des salariés de manifester leur religion sur le lieu de travail. Suivant une approche largement fondée sur la pensée des droits de l'homme de la Cour européenne des droits de l'homme (dont les résultats en matière de protection de la liberté de religion sont plutôt modestes) ${ }^{35}$, la CJUE a jugé que les politiques des entreprises interdisant le port du voile ne constituaient pas une discrimination directe fondée sur la religion, comme l'interdit la directive 2000/78. Le raisonnement et les conclusions de la Cour ont donné lieu à de nombreuses critiques ${ }^{36}$ et suscité des regrets généralisés de ne pas avoir réussi à servir à la fois les objectifs d'égalité du droit anti-discrimination et la cause de l'intégration européenne. En effet, les cas de licenciements ou de démissions de salariés appartenant à des minorités religieuses ne sont pas rares dans l'UE, comme en

33 Voir notamment I. Matzner-Heruti, op. cit. sur l'interaction problématique entre masculinité et prestation de soins; M. Saito, "Current Issues Regarding Family Caregiving and Gender Equality in Japan: Male Caregivers and the Interplay Between Caregiving and Masculinities », JLR, 2017, vol. 14,1, p. 92.

34 V. Narain, "Gender, Religion and Workplace: Reimagining Reasonable Accommodation ", CL\&ELJ, 2017, vol. 20, p. 307; L.P. Lampron, « Religious discrimination, diversity, interculturalism, accommodation: The Charter of Quebec Values and the Workplace and Competing Visions of an Inclusive Society », CL\&ELJ, 2017, vol. 20, 2, p. 339.

35 Voir E. Sychenko, op. cit., p. 121.

36 G. Busschaert, S. De Somer, "You Can Leave Your Hat on, but Not Your Headscarf: No Direct Discrimination on the Basis of Religion », IJCCLLIR, 2017, 4, p. 553; F. Dorrsemont, " Freedom of religion in the workplace and the Court of Justice of the European Union: A return to the principle of cuius regio, eius religio ?", Revue de Droit Comparé du Travail et de la Sécurité Sociale, 2017, English Electronic Edition, 4, p. 200; L. Vickers, Achbita et Bougnaoui, "One step forward and two steps back for religious diversity in the workplace », ELLJ, 2017, 3, p. 232. Pour différentes perspectives, voir aussi J.L. Bianco, N. Cadène, W. Wolmark, « Peut-on concevoir la neutralité dans l'entreprise ?», Revue de Droit du Travail, 2017, 4, p. 235. 
atteste une étude comparative de l'impact de ces situations sur les demandes d'allocations de chômage ${ }^{37}$.

La gestion des flux migratoires économiques et de la main-d'œuvre étrangère est un autre sujet, beaucoup plus vaste, qui implique des visions divergentes d'une société inclusive : il s'agit d'un phénomène mondial qui, comme nous l'avons déjà mentionné, s'est avéré particulièrement intéressant pour les experts en droit du travail et de la sécurité sociale.

Bien que le droit international des migrations ${ }^{38}$ favorise le respect des droits des travailleurs immigrés, force est de constater que les réglementations et pratiques nationales contredisent souvent cette tendance ${ }^{39}$. En effet, de nombreux articles ont examiné l'interaction entre les contrôles migratoires et les règles du travail, en se concentrant sur la situation spécifique de différents pays dans le monde, dont l'Australie, le Canada, la Corée du Sud et le Japon ${ }^{40}$ et, en Europe, le Royaume-Uni, I'Irlande et l'Espagne ${ }^{41}$. Cependant, l'analyse la plus approfondie a sans aucun doute porté sur l'Italie. Cet État ayant, en effet, la position géographique la plus délicate au sein de I'UE, les spécialistes de I'IALLJ ont tenté de clarifier la protection accordée aux travailleurs immigrés sur la base du principe d'égalité entre cives et non cives, en tenant compte du cadre juridique à plusieurs niveaux ${ }^{42}$. De manière générale, les différentes réglementations nationales relatives à la migration temporaire de main-d'œuvre ont beaucoup retenu l'attention ${ }^{43}$, tandis que le

37 K. Alidadi, «Religion and unemployment benefits: Comparing Belgium, the Netherlands and Great Britain », ELLJ, 2017, 1, p. 67.

38 Son utilité et ses faiblesses actuelles sont analysées par J.M. Servais, « Le droit international social des migrations ou les infortunes de la vertu », RDCTSS, 2017, 1, p. 94.

39 F. Elorza Guerrero, «The Regulation of Immigrant Labour in Spain: Ordinary Migration \& Selective Migration », E-JICLS, 2017, vol. 6, 2.

40 Voir respectivement C.F. Wright, S. Clibborn, «Back Door, Side Door, or Front Door? An Emerging De-Facto Low-Skilled Immigration Policy in Australia », CLLPJ, 2017, vol. 39, 1, p. 165; J. Fudge, J.C. Tham, " Dishing Up Migrant Workers for the Canadian Food Services Sector: Labor Law and the Demand for Migrant Workers », CLLPJ, 2017, vol. 39, p. 27; E.A. Chung, R.I. Hosoki, « Disaggregating Labor Migration Policies to Understand Aggregate Migration Realities: Insights from South Korea and Japan as Negative Cases of Immigration », CLLPJ, 2017, vol. 39, p. 83.

41 Voir respectivement C. Bales, «Immigration Raids, Employer Collusion and the Immigration Act 2016 », ILJ, 2017, 2, p. 279; C. Murphy, "Tackling Vulnerability to Labour Exploitation through Regulation: The Case of Migrant Fishermen in Ireland », ILJ, 2017, 3, p. 417; E. Guerrero, op. cit.

42 Voir surtout A. Garilli, «Immigrati e diritti sociali: parità di trattamento e tutela multilivello », DLM, 2017, 1, p.13; McBritton, "Lavoro extracomunitario, mercato del lavoro, contratti », RGL, 2017, 4, I, p. 582; S. Bologna, «Eguaglianza e welfare degli immigrati: tra self-restraint legislativo e aperture giurisprudenziali e contrattuali », RGL, 2017, 4, I, p. 63.

43 S. Ariyawansa, "A Red-Tape Band-Aid or a Solution? Lessons from the United Kingdom's Gangmasters (Licensing) Act 2004 for Temporary Migrant Workers in the Australian Horticulture Industry », AJLL, 2017, vol. 30, 2, p. 158 ; K.L. Griffith, S.M. Gleeson, "The Precarity of Temporality: How Law Inhibits Immigrant Worker Claims », CLLPJ, 2017, vol. 39, 1, p. 111; C. Janda, "We Asked for Workers... Legal Rules on Temporary Labor Migration in the European Union and Germany ", CLLPJ, 2017, 1, p. 143 ; P. Martin, " Guest or Temporary Foreign Worker Programs », CLLPJ, 2017, vol. 39, 1, p. 189; V. Papa, "Dentro o fuori il mercato? La nuova disciplina del lavoro stagionale degli stranieri tra repressione e integrazione $», D R I, 2017,2$, p. 363. 
débat scientifique n'a pas négligé la condition particulièrement vulnérable des travailleurs migrants en situation irrégulière ${ }^{44}$.

\section{II - TRANSFORMATIONS DU TRAVAIL : LES NOUVEAUX DÉFIS ET LES NOUVEAUX RISQUES « METTENT À L'ÉPREUVE » LA LÉGISLATION}

Les profonds changements sur le marché du travail - induits par l'innovation technologique et organisationnelle liée au monde numérique, ainsi que par des facteurs démographiques, géographiques et environnementaux - ont fait émerger de nouveaux modèles économiques, de nouvelles formes d'organisation du travail et des relations professionnelles inédites.

Sur cette thématique, les articles de l'IALLJ parus en 2017 abordent de nombreux sousthèmes très variés, dont certains présentent un intérêt particulier pour cette synthèse. Ils peuvent être divisés en quatre sujets principaux : la question du travail atypique et de la précarité ; la «zone grise » entre l'emploi et le travail indépendant ; le rôle de la protection du droit du travail de la « main-d'œuvre numérique » ; l'impact de l'utilisation croissante des TIC sur les droits des travailleurs.

\section{A - TRAVAIL ATYPIQUE ET PRÉCARITÉ}

En 2017, un certain nombre d'articles parus dans des revues membres de l'IALLJ ont abordé la question du travail atypique et de la précarité, thématique largement étudiée dans les synthèses des années passées.

Comme nous le savons, le contrat de travail classique (défini comme un accord stable, à durée indéterminée, et direct entre un salarié à temps plein et son employeur unique) a toujours servi de modèle pour réglementer le travail, alors que les formes atypiques de travail font figures d'exception à cette norme. À ce titre, ces dernières ont souvent fait l'objet d'un traitement différent: le débat sur la manière d'adapter la réglementation existante pour faire face à l'augmentation et à l'expansion de la main d'œuvre atypique dans le monde s'est donc poursuivi dans les revues de l'IALLJ parues en 2017, aussi bien sur le plan général ${ }^{45}$ que sur des thèmes plus spécifiques touchant à la sécurité sociale et aux droits collectifs. En ce qui concerne les garanties de sécurité sociale, différentes formes flexibles de travail peuvent désavantager les travailleurs : soit pour avoir droit aux prestations sociales, car leur instabilité peut les empêcher d'atteindre la période minimale requise pour accéder à certaines prestations telles que les allocations chômage ou une pension complète, soit parce que leurs salaires inférieurs et progressant lentement génèrent des prestations de

44 L. Calafà, "Lavoro irregolare (degli stranieri) e sanzioni. II caso italiano », LD, 2017, 1, p. 67; C. Faraldo Cabana, «Emplear a ciudadanos extranjeros o menores sin permito de trabajo: un nuevo delito contra los derechos de los trabajadores? ", RDS, 2017, 78, p. 127; F. Monereo Pérez, Vila Tierno, "La (des)protección social del trabajador extranjero en situación irregular. La incidencia de las autorizaciones administrativas previas respecto al reconocimiento de la prestación por desempleo », DRL, 2017, 11, p. 1073.

45 M.E. Casas Baamonde, "Precariedad del trabajo y formas atípicas de empleo, viejas y nuevas ¿Hacia un trabajo digno ? ", DRL, 2017, 9, p. 867; A.O. Goldín, " Huida, desestandarización y debilitamiento subjetivo del derecho del trabajo. Ensayo sobre un itinerario », RDL, 2017, 10, p. 977; T. Treu, « Una seconda fase della flexicurity per l'occupabilità », DRI, 2017, 3, p. 597. 
bas niveau ${ }^{46}$. S'agissant par ailleurs de la réglementation des droits collectifs, bon nombre des limitations et restrictions à la liberté d'association, au droit de négociation collective et au droit de grève (telles que les interdictions antitrust sur la négociation collective, les votes de grève obligatoire, la distinction entre grève politique et économique, etc.) impactent de manière disproportionnée les travailleurs atypiques, menaçant ainsi l'exercice effectif des droits collectifs. À ce propos, il a été avancé que les restrictions et les limites aux droits collectifs devaient être révisées pour garantir leur compatibilité avec une approche davantage fondée sur les droits humains ${ }^{47}$.

Concernant les relations de travail atypiques, les spécialistes de I'IALLJ ont le plus souvent examiné les contrats à durée déterminée d'une part, et le travail intérimaire d'autre part (à l'exception du travail de plateforme qui sera examiné ci-dessous, au § 1.4). Certains auteurs se sont également penchés sur d'autres formes d'emploi atypique, comme le travail saisonnier ${ }^{48}$, les " contrats zéro heure » ou le travail occasionnel au Royaume-Uni ${ }^{49}$ et en Slovénie ${ }^{50}$, les contrats $d^{\prime}$ « association à participation » en Italie ${ }^{51}$, ainsi que certaines formes traditionnelles de travail précaire comme le travail domestique en Espagne ${ }^{52}$. S'agissant de l'emploi à durée déterminée, la plupart des articles ont été publiés dans des revues espagnoles ou dans des revues qui présentent un intérêt pour toute l'UE.

Tout d'abord, certains des articles examinés analysaient l'accord-cadre sur le travail à durée déterminée, transposé par la suite dans la directive 1999/70 du Conseil. Ils étudiaient la jurisprudence de la CJUE ${ }^{53}$, afin notamment de déterminer si la directive avait atteint son objectif visant à rapprocher les législations nationales sur les contrats de travail à durée déterminée successifs dans les États membres de l'UE ${ }^{54}$. À cet égard, l'étude comparative de Kamanabrou portant sur quinze États membres a révélé que, malgré quelques différences notables dans les détails, le niveau de protection dans les États membres reste dans une large mesure comparable. Néanmoins, la protection contre l'abus de contrats à durée déterminée successifs est encore assez faible, en raison du fait que l'accord-cadre ne peut offrir de protection aux travailleurs que dans les limites de ses dispositions.

46 Voir P. Schoukens, A. Barrio, «The changing concept of work: When does typical work become atypical », ELLJ, 2017, 4, p. 306, qui traite de la nécessité d'adapter les principes de base de la sécurité sociale aux caractéristiques particulières du travail atypique.

47 V. De Stefano, "Non-Standard Work and Limits on Freedom of Association: A Human RightsBased Approach », ILJ, 2017, vol. 46, 2, p. 185.

48 Surtout des travailleurs migrants non ressortissants de I'UE, voir V. Papa, op. cit. ; M.E. ZoeteweijTurhan, "The Seasonal Workers Directive», ELLJ, 2017, 1, p. 28 ; C. Janda, op. cit.

49 A. Sanders, "Fairness in the Contract of Employment », ILJ, 2017, vol. 46, 4, p. 508; A.C.L. Davies, "Getting More Than You Bargained for? Rethinking the Meaning of 'Work' in Employment Law », ILJ, 2017, vol. 46, 4, p. 477.

50 N. Scortegagna Kavčnik, «Placilo in drugi pravni vidiki študentskega dela », E\&E, 2017, 2/3, p. 243.

51 A. Allamprese, "L'associazione in partecipazione con associato d'opera: un tipo contrattuale 'sospetto' », LD, 2017, 2, p. 325.

52 G. García González, « Derechos sociales y empleados del hogar: reformas jurídicas inaplazables para la dignificación del trabajo doméstico en España », RDS, 2017, 77, p. 83; E. García Testal, « La necesidad de una protección por desempleo para los trabajadores domésticos en España », RDS, 2017, 79, p. 93.

53 S. Krebber, « Die unionsrechtlichen Vorgaben zur Zulässigkeit der Befristung von Arbeitsverhältnissen », EuZA, 2017, 1, p. 22.

54 S. Kamanabrou, "Successful Rules on Successive Fixed-Term Contracts? », ICLLIR, vol. 33, 2017, 2, p. 221. 
En outre, dans les revues espagnoles, le débat s'est concentré sur le règlement concernant les contrats à durée déterminée actuellement en vigueur en Espagne (où le taux de travail temporaire est très élevé avec 26,3 \% en 2016), soulignant dès lors les faiblesses de ce règlement et réclamant qu'il soit urgemment réformé ${ }^{55}$. La loi ne fait aucune distinction, alors qu'elle devrait le faire, entre les contrats temporaires qui répondent à des besoins saisonniers et conjoncturels réels du système de production, et ceux qui sont utilisés de façon injustifiée, frauduleuse ou abusive, pour une plus grande souplesse contractuelle ${ }^{56}$. A cet égard, plusieurs auteurs se sont intéressés à l'arrêt de la CJUE dans l'affaire De Diego Porras et à sa "suite » en Espagne. La Cour de justice a déclaré que la distinction entre le régime de résiliation des contrats de remplacement temporaire et celui des contrats à durée indéterminée est discriminatoire, dans la mesure où l'indemnisation n'est prévue que pour ces derniers. Ce jugement a provoqué un véritable «tsunami ${ }^{57}$ dans la doctrine espagnole et a soulevé la question de savoir si la décision De Diego Porras s'appliquait à d'autres arrangements contractuels temporaires ${ }^{58}$.

Par ailleurs, tout au long de l'année 2017, les revues de l'IALLJ ont également consacré un espace au développement de l'emploi à durée déterminée en lien avec certains systèmes juridiques ${ }^{59}$ ou catégories spécifiques de travailleurs, tels que les cadres publics et privés ${ }^{60}$, les travailleurs du bâtiment ${ }^{61}$ et les chercheurs ${ }^{62}$.

Enfin, concernant le travail intérimaire, plusieurs auteurs ont présenté des réflexions détaillées sur la réforme allemande entrée en vigueur en $2017^{63}$, remettant en question sa conformité avec la directive 2008/104 relative au travail intérimaire et relevant des aspects critiques, comme la possibilité pour les partenaires sociaux de ne pas appliquer la période maximale d'intérim ainsi que la faculté de déroger au principe fondamental

55 T. Sala Franco, E. López Terrada, «Propuestas para un debate sobre la reforma de la contratación temporal », DRL, 2017, 11, p. 1090.

56 M.E. Casas Baamonde, "La contratación temporal: problemas y soluciones. Un debate necesario ", DRL, 2017, 11, p. 1098; A. Goldin, op. cit.

57 T. Sala Franco, "Acerca de la Directiva comunitaria 1999/70, sobre el trabajo de duración determinada y de la sentencia del Tribunal de Justicia comunitario de 14 de septiembre de $2016 », D R L, 2017,3$, p. 217.

58 A. López Hernández, «La contratación laboral temporal a partir del caso de Diego Porras (asunto C-596/2014)», DRL, 2017, 10, p. 998.

59 Voir par exemple, M. Debono, V. Marmarà, "Perceived Precarious Employment in Malta », E-JICLS, 2017, vol. 6, 2.

60 P. Monda, «Il lavoro a tempo determinato del dirigente privato e pubblico », DRI, 2017, 4, p. 1081.

61 C. Dechristé, «Le contrat de chantier ou d'opération: le grand retour?», Revue de Droit du Travail, 2017, 10, p. 633.

62 M.D. Ferrara, "La ricerca a termine: problemi e prospettive del reclutamento dei ricercatori universitari », DLM, 2017,1, p. 61.

63 W. Hamann, E. Klengel, "Die Überlassungsdauer des reformierten AÜG im Lichte des Unionsrechts », EuZA, 2017, 2, p. 194; W. Hamann, E. Klengel, «Die AÜG-Reform 2017 im Lichte der Richtlinie Leiharbeit », EuZA, 2017, 4, p. 485; M. Maul-Sartori, P. Remy, "La réforme de la loi allemande relative au prêt de main-d'œuvre: des rapprochements avec le droit français, notamment à la faveur de la transposition de la directive 2008/104 ? ", Revue de Droit du Travail, 2017, 2, p. 148 ; J. Ulber, " Die AÜG-Reform oder besser: Neuregelung zur Diskriminierung und zum funktionswidrigen Einsatz von Leiharbeitnehmern », AUR, 2017, 6, p. 238. 
d'égalité de traitement entre les travailleurs intérimaires et ceux titulaires d'un contrat à durée indéterminée ${ }^{64}$.

\section{B - ENTRE EMPLOI SALARIÉ ET TRAVAIL INDÉPENDANT}

De toute évidence, « en raison de la complexité des modalités de travail se situant dans la «zone grise», entre le travail salarié et le travail indépendant, il devient de plus en plus difficile de déterminer si une personne qui travaille relève du " salariat " ${ }^{65}$.

À ce sujet, de nombreux articles parus dans les revues de 2017 de l'IALLJ ont examiné la législation et la jurisprudence nationales de nombreux pays du monde.

La réglementation italienne de l'emploi et du travail indépendant a suscité beaucoup d'intérêt, en particulier dans les revues italiennes où les articles portaient principalement sur les réformes législatives introduites en 2015 et 2017. La réforme de 2015 a étendu la protection jusqu'alors accordée au travail dépendant classique au «travail organisé par l'employeur ${ }^{66}$, tandis qu'une nouvelle loi sur le travail indépendant adoptée en 2017 a introduit de nouvelles mesures de protection pour les entrepreneurs indépendants ; mesures désormais profondément ancrées dans le droit des contrats (Perulli appelle cette réforme « le retour du travail indépendant dans le giron du droit des contrats $»^{67}$ ).

La réglementation allemande a également été étudiée, afin d'analyser la situation juridique des "entrepreneurs individuels " (c'est-à-dire des entrepreneurs de plus en plus nombreux qui n'emploient personne) et de déterminer les moyens permettant de leur assurer une meilleure protection. Plus généralement, il a été question d'examiner la notion de salarié au sens du § 611a BGB tel que modifié en 2017 et la tentative ratée de faire figurer dans le Code civil allemand les premiers indicateurs qui seraient à la disposition des juridictions pour établir l'existence d'une relation de travail68. Le statut de salarié est un sujet particulièrement sensible dans la plupart des pays. Au Royaume-Uni par exemple, des études intéressantes ont abordé le problème de la « disponibilité », c'est-à-dire lorsque les travailleurs ne sont pas activement engagés dans des tâches purement professionnelles

64 J. Uber, ibid. Sur la mise en œuvre du principe de l'égalité de traitement, voir également B. Kresal Šoltes, "Razmejitev obveznosti med agencijo in podjetjem uporabnikom ter načelo enakega obravnavanja - je lahko model tudi za druge nestandardne oblike dela », E\&E, 2/3, p. 199.

65 P. Schoukens, A. Barrio, op. cit., 2017, p. 309.

66 F. Martelloni, "I rimedi nel 'nuovo' diritto del lavoro autonomo », LD, 2017, 3/4, p. 517; S. Bini, «Para-subordinación y autonomía. El derecho del trabajo italiano en transformación », TL, 2017, 136, p. 49; D. Mezzacapo, "L'incerta figura delle collaborazioni organizzate dal committente », RGL, 2017, 1, I, p. 49; A. Riccobono, "Sulla 'Carta dei diritti' della Cgil. La riforma dei contratti e dei rapporti di lavoro: privato e pubblico a confronto », RGL, 2017, 1, I, p. 63.

67 A. Perulli, « Il lungo viaggio del lavoro autonomo dal diritto dei contratti al diritto del lavoro, e ritorno ", LD, 2017, 2, p. 25. Voir aussi, S. Giubboni, " Il Jobs act del lavoro autonomo: commento al capo I della legge n. 81/2017 », DLRI, 2017, 155, p. 471; Marteloni, op. cit.; G. Santoro-Passarelli, "Lavoro etero-organizzato, coordinato, agile e telelavoro: un puzzle non facile da comporre nell'impresa in via di trasformazione $», D R I, 2017,3$, p. 771.

68 B. Waas, "What role for solopreneurs in the labour market? », ELLJ, 2017, 2, p. 154; R. Wank, "Der Arbeitnehmer-Begriff im neuen § 611a BGB», AUR, 2017, 4, p. 140. 
mais ne sont pas non plus complètement disponibles ${ }^{69}$. En Espagne, une réforme concernant le travail indépendant a été introduite en 2017 et a généré une réglementation plutôt contradictoire ${ }^{70}$.

La question se pose pareillement aux États-Unis : Treu, par exemple, a analysé la jurisprudence américaine concernant le statut des travailleurs des entreprises relevant de l'économie de plateforme (voir ci-dessous, § 1.3), faisant valoir que l'approche corrective adoptée par la Common law peut également être intéressante pour d'autres juges et législateurs, dans la mesure où elle permet une meilleure identification des protections et droits applicables à ces travailleurs « de plateformes $»^{71}$ par rapport à la classification traditionnelle. À cet égard, le fait que les plateformes offrent aux entreprises l'avantage de pouvoir diviser les tâches permanentes en «microtâches", susceptibles d'être proposées à un nombre indéfini de parties intéressées sur Internet, pourrait entraîner très prochainement une nouvelle augmentation du travail indépendant ${ }^{72}$. La plupart de ces travailleurs resteront précaires et vulnérables. Ce qui est nouveau dans l'économie de plateforme, c'est le développement de technologies qui permettent aux entreprises de " prétendre ne pas employer ceux qui travaillent pour elles » et de créer dès lors " un pseudo marché du travail où les travailleurs seraient des indépendants recevant du travail et fournissant des services via une plateforme numérique créée par la société " $^{73}$. Cependant, ces travailleurs ont encore besoin de protection et, tout au long de l'année 2017, de nombreux auteurs ont réfléchi à la manière dont le droit du travail pourrait répondre à cette nécessité.

\section{C - TRAVAILLER SUR LES PLATEFORMES À L'ÈRE DU NUMÉRIQUE}

Toutes les revues de l'IALLJ ont étudié, de manière approfondie, le rôle de la protection offerte par le droit du travail à l'égard de la " main-d'œuvre numérique », au caractère particulièrement hétérogène, issue de la nouvelle économie (indifféremment dénommée économie " de plateforme ", "à la tâche ", " de partage ", "à la demande " ou encore "collaborative »), dans laquelle les plateformes numériques sont de plus en plus utilisées pour effectuer ou organiser le travail. Les spécialistes manifestent en effet un intérêt croissant pour l'évolution du droit du travail dans l'économie de plateforme et, dans de nombreux articles et plusieurs numéros spéciaux de revues de l'IALLJ (tels RGL en Italie

69 A.C.L Davies, op. cit. Voir aussi M. Böttcher, «Der Employment Status im englischen Arbeitsrecht», EuZA, 2017, 3, p. 370. Sur la question du statut professionnel des membres d'une société à responsabilité limitée (SARL), E. Berry, "When Is a Partner/LLP Member Not a Partner/LLP Member? The Interface with Employment and Worker Status », ILJ, 2017, vol. 46, 3, p. 309.

70 C.L. Alfonso Mellado, G. Fabregat Monfort, R. Pardo Gabaldón, «Reformas urgentes en materia de trabajo autónomo: medidas laborales », RDS, 2017, 80, p. 27. Voir aussi A. Todolí-Signes, «Los falsos autónomos en el contrato de franquicia », RDS, 2017, 77, p. 105 et A. Duval, O. van Maren, «The Labour Status of Professional Football Players in the European Union. Unity in/and/ or diversity? », ELLJ, 2017, 3, p. 258.

71 T. Treu, «Rimedi, tutele e fattispecie: riflessioni a partire dai lavori della Gig economy », LD, 2017, $3 / 4$, p. 367.

72 B. Waas, op. cit.

73 M. Sargeant, "The Gig Economy and the Future of Work », E-JICLS, 2017, vol. 6, 2; A. TodolíSignes, "The End of the Subordinate Worker? The On-Demand Economy, the Gig Economy, and the Need for Protection for Crowdworkers », IJCLLIR, 2017, vol. 33, 2, p. 241. 
et TL en Espagne), ils s'interrogent sur les possibilités offertes par le droit du travail pour repenser ses frontières et adapter sa protection au défi de «l'ubérisation $»^{74}$.

Dans le monde entier sont survenus des litiges relatifs à la qualification de la relation de travail des travailleurs de l'« économie à la tâche $»^{75}$. II n'est donc pas surprenant de constater que de nombreux articles parus dans les revues de I'IALLJ ont traité des récents procès intentés contre le géant Uber par ses chauffeurs, procès qui ont donné lieu à des jugements intéressants (en particulier par des tribunaux au Royaume-Uni, aux États-Unis et par la CJUE) ${ }^{76}$.

Pour le droit du travail, il convient de se demander si les travailleurs de plateformes sont considérés comme des salariés ${ }^{77}$ et si les plateformes peuvent dès lors être tenues pour responsables en tant qu'employeurs ${ }^{78}$. Cette question a également été abordée par la Commission européenne, dans une Communication intitulée « Un agenda européen pour l'économie collaborative » qui alerte sur le fait que les modalités de travail plus flexibles générées par l'économie collaborative créent, de fait, une incertitude sur les droits applicables et sur la protection sociale. Néanmoins, les orientations proposées par cette Communication aux États membres de l'UE (en particulier sur la manière dont la distinction traditionnelle entre travailleurs indépendants et travailleurs salariés s'applique dans l'économie collaborative) se sont au final avérées inutiles ${ }^{79}$.

Par ailleurs, les tribunaux et législateurs chinois se sont penchés sur des questions plus ou moins pertinentes afin de déterminer le statut des chauffeurs dans le secteur des VTC. C'est ainsi qu'un spécialiste suggérait de rechercher une solution en s'appuyant sur les critères actuels de qualification de la relation de travail déjà utilisés par le droit chinois plutôt que de créer de nouvelles catégories juridiques de travailleurs ${ }^{80}$. Des études sur la répartition des risques entre les plateformes et les travailleurs des plateformes ont également attiré l'attention et ont conduit à proposer que les droits sociaux des travailleurs

74 A. Fabre, M.C. Escande-Varniol, « Le droit du travail peut-il répondre aux défis de l'ubérisation? ", RDT, 2017, 3, p. 166. Pour une perspective générale des relations de travail sur ces changements majeurs, voir Villalon, op. cit.

75 S. González Ortega, "Trabajo asalariado y trabajo autónomo en las actividades profesionales a través de las plataformas informáticas», TL, 2017, 138, p. 85 ; T. Pasquier, "Sens et limites de la qualification de contrat de travail »,RDT, 2017, 2, p. 95 ; M. Sargeant, op. cit. ; L. Ratti, "Online Platforms and Crowdwork in Europe: A Two-Step Approach to Expanding Agency Work Provisions », CLLPJ, 2017, vol. 38, 2, p. 477.

76 S. Auriemma, "Impresa, lavoro e subordinazione digitale al vaglio della giurisprudenza », $R G L$, 2017, 2, I, p. 281; V. De Stefano, "Lavoro 'su piattaforma' e lavoro non standard in prospettiva internazionale e comparata »,RGL, 2017, 2, I, p. 241; J. Prassl, " Uber devant les tribunaux », RDT, 2017, 6, p. 439 ; F. Trillo Párraga, « Uber, ¿sociedad de la información o prestadora de servicios de transporte?", RDS, 2017, 80, p. 127.

77 A. Perulli, "Lavoro e tecnica al tempo di Uber », RGL, 2017, 2, I, p. 195; A. Todolí-Signes, op. cit.; V. De Stefano, op. cit.

78 J. Prassl, M. Risak, "Le piattaforme di lavoro on demand come datori di lavoro », RGL, 2017, 2, I, p. 219.

79 A. Perulli, « II lungo viaggio del lavoro autonomo dal diritto dei contratti al diritto del lavoro, e ritorno », LD, 2, p. 25. Voir aussi L. Ratti op. cit. ; M.E. Casas Baamonde, op. cit. ; M. Rodríguez-Piñero Royo, "La agenda reguladora de la economía colaborativa: aspectos laborales y de seguridad social », TL, 2017, 138, p. 125.

80 M. Zou, "The Regulatory Challenges of 'Uberization' in China: Classifying Ride-Hailing Drivers », IJCLLIR, 2017, vol. 33, 2, p. 269. Pour les États-Unis, voir T. Treu, op. cit. 
exerçant leurs activités via des plateformes numériques soient déterminés sur la base du degré d'exposition aux risques, indépendamment de la qualification donnée par les parties au contrat ${ }^{81}$. D'autres questions enfin, relatives au cadre juridique des relations professionnelles, de la négociation collective et de la grève ${ }^{82}$, ou encore au rôle potentiel des syndicats, ont été mises en exergue ${ }^{83}$.

\section{D - TIC ET DROITS DES TRAVAILLEURS : DROIT À LA DÉCONNEXION ET DROIT À LA VIE PRIVÉE}

L'innovation technologique impacte de plus en plus le « monde du travail » dans ses aspects aussi divers et variés que le temps et le lieu de travail, la santé et la sécurité, les instruments de contrôle de l'employeur, etc ${ }^{84}$. Ainsi, les articles qui, d'un point de vue différent, étudient l'impact des technologies de l'information et de la communication sur deux droits des travailleurs (le droit à la déconnexion d'une part, et le droit à la vie privée d'autre part) seront examinés ci-dessous.

S'agissant du droit des salariés à la déconnexion, c'est-à-dire le droit d'être indisponible en dehors des heures normales de travail, il n'est pas étonnant que la majorité des articles de I'IALLJ aient traité des législations française et italienne, toutes deux récemment adoptées sur le sujet. Le droit à la déconnexion, introduit en France le $1^{\text {er }}$ janvier 2017 par la Loi Travail (modifiant le Code du travail) qui obligeait les entreprises d'une certaine taille à négocier le droit à la non disponibilité avec les syndicats représentatifs, a ainsi fait l'objet d'une analyse détaillée dans quelques articles intéressants ${ }^{85}$. En outre, des comparaisons ont été opérées avec la législation italienne de 2017 sur le «lavoro agile » ou « travail intelligent »c'est-à-dire le travail en partie effectué en dehors des locaux de l'employeur au moyen d'outils technologiques - qui, plus que les formes traditionnelles de travail, soulève la question de la distinction entre temps de travail et périodes de repos, et s'interroge donc sur le droit des travailleurs à la déconnexion ${ }^{86}$.

S'agissant par ailleurs du droit à la vie privée, les innovations technologiques peuvent également affecter les droits inaliénables de la personne humaine, notamment ceux relatifs à la vie privée et à la dignité du salarié. Avec le recours croissant des entreprises aux nouvelles technologies, force est de constater que le travailleur est potentiellement soumis

81 P. Loi, « Il lavoro nella Gig economy nella prospettiva del rischio », RGL, 2017, 2, I, p. 259.

82 M. Faioli, « Jobs 'App', Gig economy e sindacato », RGL, 2017, 2, I, p. 291.

83 S. Engblom, "Una prospettiva sindacale su digitalizzazione e Gig economy ", RGL, 2017, 2, I, p. 357; M. Mensi, «Lavoro digitale e sindacato », RGL, 2017, 3, I, p. 525.

84 C. Sánchez-Rodas Navarro, "Poderes directivos y nuevas tecnologías », TL, 2017, 138, p. 163; J. Cruz Villalon, «El futuro del trabajo y su gobernanza », TL, 2017, 137, p. 13.

85 P.H. Cialti, «El derecho a la desconexión en Francia: ¿más de lo que parece ? », TL, 2017, 137, p. 163 ; E. Durlach, M. Renaud, "Das Recht auf Nichterreichbarkeit - Droit à la Déconnexion nach der Loi Travail », AUR, 2017, 5, p. 196 ; C. Molina Navarrete, « Jornada laboral y tecnologías de la info-comunicación: 'desconexión digital', garantía del derecho al descanso », TL, 2017, 138, p. 249; D. Senčur Peček, « Delovni čas v dobi stalne dosegljivosti », E\&E, 2017, 2/3, p. 155.

86 Voir notamment le numéro spécial de DRI sur le "lavoro agile ». E. Dagnino, "II diritto alla disconnessione nella legge $n^{\circ} 81 / 2017$ e nell'esperienza comparata », DRI, 2017, 4, p. 1024. Voir aussi A. Allamprese, F. Pascucci, "La tutela della salute e della sicurezza del lavoratore 'agile' », $R G L, 2017,2$, I, p. 307; R. Casillo, "La subordinazione 'agile' », DLM, 2017, 3, p. 529. 
à un contrôle constant, délocalisé, sans discrimination et toujours plus envahissant ${ }^{87}$. Les spécialistes de I'IALLJ ont accordé une attention particulière à l'arrêt de la Grande Chambre de la Cour européenne des droits de l'homme dans l'affaire Bărbulescu II c. Roumanie, rendu en septembre 2017, dans lequel la Cour de Strasbourg, saisie d'un litige concernant le contrôle par un employeur privé des emails d'un de ses salariés, a invoqué une violation de l'article 8 de la Convention, rompant ainsi avec sa jurisprudence de $2016^{88}$. Plusieurs auteurs de revues espagnoles de l'IALLJ en particulier, ont confronté la jurisprudence de la Cour européenne des droits de l'homme à celles de la Cour suprême et de la Cour constitutionnelle espagnoles, notamment en ce qui concerne la portée du droit à l'information des salariés ${ }^{89}$. Parallèlement, d'autres auteurs ont exprimé le souhait qu'en matière de protection des données personnelles, la législation et la jurisprudence espagnoles respectent le Règlement général sur la protection des données de I'UE, entré en vigueur le 25 mai $2018^{90}$. Dans d'autres pays également, comme en Italie et aux États-Unis ${ }^{91}$, la question de la surveillance des travailleurs sur le lieu de travail s'avère très problématique en raison des intérêts en jeu diamétralement opposés et de la difficulté à assurer une protection adéquate de tous les travailleurs dans le cadre d'une relation de travail profondément inégale.

\section{III - PERSPECTIVES EN MATIÈRE DE DROIT COLLECTIF DU TRAVAIL}

Comme les années précédentes, les revues de l'IALLJ en 2017 ont porté une grande attention au droit collectif du travail. Toutes ces publications ont pour point commun une approche historique. En effet, les présentations remontent parfois loin dans le temps, comme les premières recherches universitaires sur le droit du travail australien : 1920196092. La revue AuR a même créé une rubrique spéciale, intitulée «Travail et historique du droit » et publiée tous les deux mois, avec des articles comme : les grèves dans la fonction publique ${ }^{93}$; I'Arbeit-Nordwest et METALL NRW (association des employeurs) ${ }^{94}$;

87 M.J. Cervilla Garzón, «Efectos del uso de la aplicación 'whatsapp' en el marco de las relaciones laborales », TL, 2017, 136, p. 73; C. Colapietro, "Tutela della dignità e riservatezza del lavoratore nell'uso delle tecnologie digitali per finalità di lavoro », DLRI, 2017, 155, p. 439.

88 R. Gallardo Moya, «Un límite a los límites de la vida privada y de la correspondencia en los lugares de trabajo », RDS, 2017, 79, p. 141 ; B. Ancel, « Big Brother au bureau : impératif sécuritaire ou crépuscule du droit à la vie privée? Regards croisés États-Unis - Europe », RDT, 2017, 3, p. 219.

89 M.E. Terradillos Ormaechea, «El principio de proporcionalidad como referencia garantista de los derechos de los trabajadores en las últimas sentencias del TEDH dictadas en materia de ciberderechos », RDS, 2017, 80, p. 139 ; F. Valdés Dal-Ré, " Doctrina constitucional en materia de videovigilancia y utilización del ordenador por el personal de la empresa », RDS, 2017, 79, p. 15 ; E. González Biedma, «Derecho a la información y consentimiento del trabajador en materia de protección de datos », TL, 2017, 138, p. 223.

90 J. Goñi Sein, « Nuevas tecnologías digitales, poderes empresariales y derechos de los trabajadoresanálisis desde la perspectiva del Reglamento Europeo de Protección de Datos de $2016 », R D S, 2017,78$, p. 15.

91 Voir respectivement, V. Aniballi, « La regulación italiana de los controles a distancia: el 'nuevo' art. 4 del Estatuto de los trabajadores», DRL, 2017, 8, p. 795 ; et B. Ancel, op. cit.

92 R. Naughton, "Early academic research in Australian labour law: 1920-60 », AJLL, 2017, vol. 30, 1, p. 58.

93 B. Keller, « Arbeit und Rechtsgeschichte, Die großen Streiks im öffentlichen Dienst, Verlauf und Erklärung », AuR, 2017, 1, p. G 1.

94 L. Mallmann, « Arbeit-Nordwest und METALL NRW, Kontinuität oder Diskontinuität? », AuR, 2017, 3, p. G 5 . 
la Loi Wagner (US) ${ }^{95}$; l'émergence de l'assurance accident obligatoire ${ }^{96}$; I'histoire des lois sur la promotion de l'emploi ${ }^{97}$; la grève générale à Mössingen du 31 janvier $1933^{98}$. Dans d'autres revues, nombre d'articles ont mis en lumière d'importants jugements et évolutions survenus au cours des vingt dernières années. A titre d'exemples, nous citerons l'arrêt de la Cour de Strasbourg Svenska Transportarbetareförbundet et Seko c. Suède ${ }^{99}$; les leçons de la grève 2009-2010 de la section locale 6500 des Métallos à Sudbury, Ontario ${ }^{100}$; l'impact de la Loi sur les conflits industriels revisité : perspective sur l'évolution des deux dernières décennies ${ }^{101}$; la reconnaissance du droit de négocier collectivement en Australie et au Royaume-Uni ${ }^{102}$; la réforme du droit du travail grec par les Mémorandums I, II et III ${ }^{103}$.

En 2017, des questions et des défis similaires se sont posés dans le monde entier. Fréquemment, les changements structurels dans la représentation des travailleurs ont suscité des discussions académiques et contribué à mettre en place de nouvelles stratégies syndicales, dans un environnement politique et professionnel en perpétuelle mutation. De nombreux articles, en particulier dans des revues spécialisées d'Europe du Sud, ont eu tendance à se concentrer sur les questions structurelles de la négociation collective, comme l'évolution des formes ou du contenu des accords collectifs de travail. II s'agissait de réagir, d'une part aux conséquences de l'intervention législative et, d'autre part, aux changements de l'environnement social et des formes de production. Un débat mondial s'est alors engagé sur les diverses formes d'action syndicale et les garanties du droit de grève. Si l'autonomie de la négociation collective et le droit de grève ont certes subi des pressions politiques considérables, de nouvelles perspectives se sont dessinées, à la faveur notamment d'un recours accru au répertoire international des droits humains et du droit comparé.

95 T.C. Kohler, «Der Wagner Act », AuR, 2017, 5, p. G 9.

96 M. Fuchs, " Ein sozialrechtlicher Quantensprung: Die Entstehung der gesetzlichen Unfallversicherung ", AuR, 2017, 7, p. G 13.

97 R. Buschmann, «Geschichte der Beschäftigungsförderungsgesetze », AuR, 2017, 9, p. G 17

98 W. Däubler, "Der Mössinger Generalstreik vom 31.1.1933 - praktiziertes Widerstandsrecht?", AuR, 2017, 11, p. G 21.

99 K.D. Ewing, J. Hendy, "The Strasbourg Court Treats Trade Unionists with Contempt: Svenska Transportarbetareförbundet and Seko v. Sweden », ILJ, 2017, vol. 46, 3, p. 435.

100 A.D.K. King, "Memory, Mobilization and the social Basis of Intra-Union Division: Some Lessons from the 2009-2010 USW Local 6500 Strike in Sudbury, Ontario », E-JICLS, 2017, vol. 6, 3.

101 J. Elgar, B. Simpson, « The Impact of the Law on Industrial Disputes Revisited: A Perspective on Developments over the Last Two Decades », ILJ, 2017, vol. 46, 1, p. 6.

102 A. Forsyth, J. Howe, P. Gahan, I. Landau, «Establishing the Right to Bargain Collectively in Australia and the UK: Are Majority Support Determinations under Australia's Fair Work Act a More Effective Form of Union Recognition? », ILJ, 2017, vol. 46, 3, p. 335.

103 D. Sideris, C. Triadafillidis, « Die Reform des griechischen Arbeitsrechts durch Memoranda I, II und III », ZIAS, 2017, vol. 31, 1, p. 66. 


\section{A - STRUCTURES DYNAMIQUES DE LA REPRÉSENTATION COLLECTIVE DES TRAVAILLEURS}

Des réflexions approfondies ont porté sur l'applicabilité du droit collectif ${ }^{104}$, la relation entre l'individuel et le collectif ${ }^{105}$ et les nouvelles perspectives du droit du travail collectif, la reconnaissance syndicale et la négociation collective ${ }^{106}$. A l'instar du droit du travail individuel (voir ci-dessus), le droit du travail collectif est toujours à la recherche de nouvelles solutions pour les salariés précaires ${ }^{107}$ et atypiques qui sont largement privés de droits humains collectifs ${ }^{108}$. Différents points de vue ont été exposés sur la question de savoir si les législateurs devraient reconnaitre de nouvelles formes d'action collective, en dehors du contexte syndical ${ }^{109}$. Dans certains pays, les auteurs ont relevé une tendance à passer d'une convention collective interprofessionnelle à sectorielle, puis d'une convention collective de branche à une convention collective d'entreprise.

Le plus souvent, cette tendance ne s'est pas fondée sur les concepts de la négociation collective d'entreprise qui avait été débattue il y a des années pour encourager les travailleurs syndiqués de base à participer à la gestion de leurs activités. Aujourd'hui, il s'agit davantage d'une décentralisation de la négociation collective qui a pour but, d'une part de réaliser des concessions réciproques et, d'autre part, de couvrir des secteurs se trouvant jusque-là hors du champ conventionnel. Plus que des questions relatives au pluralisme syndical, il s'agit désormais de porter davantage l'attention aux questions de représentativité syndicale comme catégorie juridique ${ }^{110}$.

Le droit collectif du travail vient de sombrer dans une mer déchaînée. Les « réformes " néolibérales ont vigoureusement tenté d'influencer la structure et les résultats de la négociation collective, en essayant de porter la négociation collective à son niveau le plus inférieur. Certaines réformes fondamentales du droit du travail ont été présentées

104 M. Freedland, N. Kountouris, "Some Reflections on the 'Personal Scope' of Collective Labour Law », ILJ, 2017, vol. 46, 1, p. 52; O. Levannier-Gouël, "L'intégration étroite et permanente à la communauté de travail, Condition d'accès et de maintien des salariés dans leurs fonctions représentatives ", Revue de Droit du Travail, 2017, 1, p. 19 ; S. Banerjee, Z. Mahmood, " Judicial Intervention and Industrial Relations: Exploring Industrial Disputes Cases in West Bengal ", ILJ, 2017, vol. 46, 3, p. 366.

105 A. Bogg, «'Individualism' and 'Collectivism' in Collective Labour Law », ILJ, 2017, vol. 46, 1, p. 72.

106 K. Ewing, J. Hendy, "New Perspectives on Collective Labour Law: Trade Union Recognition and Collective Bargaining », ILJ, 2017, vol. 46, 1, p. 23.

107 A.J. Rolland, «Recent Developments in Unionizing the Precarious Workforce: The Exemption Regimes of Care Workers and Farm Workers in Quebec », CLELJ, 2017, vol. 20, 1, p. 107.

108 V. De Stefano, op. cit.

109 B. Rogers, S. Archer, "Protecting Concerted Action Outside the Union Context », CLELJ, 2017, vol. 20, 1, p. 141; D. Taras, "Nonunion Representation in Law and Practice », CLELJ, 2017, vol. 20, 1, p. 175.

110 J.I. Pérez Infante, "La estadística de convenions colectivos y la medición de la cobertura de la negociación colectiva », TL, 2017, 136, p. 159 ; De Val Tena, "El convenio colectivo de empresa », RDS, 2017, 79, p. 205. 
et analysées pour la France ${ }^{111}$, le Brésil112, I'Espagne ${ }^{113}$ et l'Italie ${ }^{114}$. En Espagne, la portée juridique des accords collectifs de travail a été amoindrie par la modification de l'art. 82 de l'Estatuto de los Trabajadores, qui autorise des dérogations aux accords collectifs à certaines conditions ${ }^{115}$. Les réformes structurelles sont généralement justifiées par la volonté d'atteindre un objectif de politique du marché du travail. Souvent néanmoins, les amendements à la loi n'ont atteint que partiellement les objectifs fixés. De nouveaux problèmes ont été mis en évidence, comme en Allemagne. Ici, la réforme controversée de la Loi sur les conventions collectives (en cas de pluralité syndicale dans un établissement, les conventions collectives d'un syndicat minoritaire ne devraient plus être applicables) est à l'origine d'une décision de la Cour constitutionnelle fédérale ${ }^{116}$ et d'un recours devant la Cour européenne des droits de l'homme dont le résultat est encore attendu.

Une série d'articles se sont par ailleurs interrogés sur la pertinence actuelle des modalités traditionnelles d'organisation collective des travailleurs ${ }^{117}$. Les exigences d'égalité ont conduit les syndicats à façonner leur politique sociale et par voie de conséquence ont élargi leur champ de responsabilité ${ }^{118}$. Face à la crise économique, en particulier dans les pays du sud de l'Europe, plusieurs auteurs ${ }^{119}$ ont donc cherché à définir les contributions que les syndicats pouvaient potentiellement apporter pour y remédier. Enfin, les syndicats ont également bénéficié de nouvelles opportunités d'information, de consultation et de présence au sein des entreprises, via le recours à des nouveaux médias électroniques ${ }^{120}$. Bien que le TFUE ait ouvert une procédure spéciale pour les négociations et les accords avec les partenaires sociaux, peu de progrès ont de fait été réalisés ces dernières années. A titre d'exemple, la Commission européenne n'a pas transmis au Conseil des ministres les accords négociés par les partenaires sociaux dans plusieurs secteurs. Dès lors, ces accords n'ont pas pu acquérir le statut de directives. Les accords autonomes passés par des partenaires sociaux au niveau européen n'ont pas la même validité dans tous les États membres. Afin de promouvoir le dialogue social, il convient donc de mettre davantage l'accent sur les résultats obtenus ${ }^{121}$.

111 A. Jeammaud, "La 'Reforma Macron' del Código del Trabajo Francés », TL, 2017, 139, p. 13.

112 J. Cavalcanti Boucinhas Filho, «La reforma laboral de 2017 y la negociación colectiva en Brasil/ The labour reform of 2017 and collective bargaining in Brazil », TL, 2017, 139, p. 159.

113 R. Bodas Martí, "Cuestiones jurisprudenciales sobre la negociación colectiva », TL, 2017, 139, p. 55 ; E. Carrizosa Prieto, « Il impacto de las normas de concurrencia tras las reformas legales », $T L$, 2017, 140, p. 75 ; M. García Jiménez, «Ámbitos de la negociación colectiva », TL, 2017, 140, p. 17.

114 U. Romagnoli, G. Cazzetta, "Sobre la crisis del derecho laboral (una entrevista)», RDS, 2017, 80, p. 13; M. Lai, « Una 'norma di sistema' per contrattazione e rappresentanza », DRI, 2017, 1, p. 45.

115 C. Sáez Lara, "Descuelgue convencional y arbitraje obligatorio »,TL, 2017, 140, p. 31.

116 K. Bepler, " Ein Zwischenurteil ? - Bemerkungen zum Tarifeinheitsurteil des Bundesverfassungsgerichts ", AuR, 2017, 10, p. 380.

117 G. Sateriale, «Ripensare la contrattazione », DRI, 2017, 3, p. 710.

118 D. Gagne, M.J. Dupuis, "Constitutionnalisation du droit du travail et transformation du devoir de représentation syndicale : quelques questionnements concernant les clauses 'orphelin' ", CLELJ, 2017, vol. 20, 1, p. 1.

119 L. Valente, "I ruoli del sindacato e delle istituzioni per la soluzione delle crisi occupazionali », DRI, 2017, 3, p. 729; A. Baylos Grau, « Notas sobre la regulación de la huelga en los servicios esenciales en Castilla-La Mancha », RDS, 2017, 78, p. 195.

120 F. Navarro Nieto, «El ejercicio de la actividad sindical a través de las tecnologías de la información y de las comunicaciones », TL, 2017, 138, p. 49.

121 V. Franca, «Bodo od evropskega socialnega dialoga ostali samo še nezavezujoči dogovori? Pregled in analiza obstoječih praks », E\&E, 2017, 4, p. 475. 
Seuls quelques pays disposent d'une "Constitution sociale de l'entreprise » dans l'entreprise, à l'instar de celle établie en Allemagne qui est associée au droit des comités d'entreprise à la co-détermination. Le sentiment généralisé d'un partenariat social cordial a déjà été mis à mal par les nombreux litiges opposant les comités d'entreprise aux employeurs en ce qui concerne la portée et l'exercice du droit à la co-détermination. Certains articles ont retracé ces débats et permis d'apporter un éclairage critique sur des sujets tels que : Droit et devoirs généraux du comité d'entreprise ${ }^{122}$; co-détermination du comité d'entreprise sur le temps de travail123; accords sur le temps de travail ${ }^{124}$; loi sur les comités d'entreprise européens - statu quo et évolution ${ }^{125}$; amendements à la loi sur les Comités d'entreprise européens concernant les marins - participation aux réunions du comité d'entreprise par vidéoconférence ${ }^{126}$. A ce titre, Thannisch ${ }^{127}$ donne un aperçu de la co-détermination au niveau du conseil d'administration.

Nonobstant les différences entre les législations nationales, quelques articles ont abordé des sujets avec un spectre disciplinaire plus large que celui des revues ici examinées, tel le Protocole additionnel à la Charte sociale européenne sur les réclamations collectives, signé le 9 novembre 1995 et ratifié par la plupart des États européens, étudié par Lukas ${ }^{128}$ qui a décrit le mécanisme de réclamation collective en question ${ }^{129}$. Outre les procédures judiciaires habituelles, d'autres voies procédurales ont également été abordées dans les articles, à savoir l'arbitrage de griefs ${ }^{130}$ ou le règlement extrajudiciaire des conflits de travail ${ }^{131}$. L'intervention judiciaire et les relations industrielles au Bengale-Occidental ont été présentées par Banerjee, Mahmood ${ }^{132}$. Par ailleurs, Rataj ${ }^{133}$ a comparé une sélection des meilleures pratiques des Cours suprêmes des États membres de l'UE et étudié leurs possibles applications dans les conflits sociaux.

122 H. Zimmermann, «Zum Stellenwert der allgemeinen Aufgaben des Betriebsrats gemäß § 80 Abs. 1 BetrVG und seinen sich daraus ergebenden Handlungspflichten », AuR, 2017, 5, p. 192.

$123 \mathrm{M}$. Eylert, "Mitbestimmung des Betriebsrats bei der Arbeitszeit im Spiegel der aktuellen Rechtsprechung", AuR, 2017, 1, p. 4.

$124 \mathrm{M}$. Halgmann, "Betriebsvereinbarungen zur Arbeitszeit - Die Rolle von Macht in Verhandlungsprozessen », AuR, 2017, 3, p. 106.

125 V. Zu Dohna-Jaeger, « 20 Jahre EBRG - Status quo und Weiterentwicklung », AuR, 2017, 5, p. 194.

126 R.P. Hayen, «Änderung des EBRG für Seeleute - Sitzungsteilnahme per Videokonferenz möglich!», AuR, 2017, 10, p. 394.

127 R. Thannisch, «Unternehmensmitbestimmung: Aktuelle Herausforderungen und Reformoptionen», AuR, 2017, 11, p. 480.

128 K. Lukas, "Der Kollektivbeschwerdemechanismus der Europäischen Sozialcharta - aktuelle Entwicklungen », ZIAS, 2017, vol. 31, 1, p. 113.

129 R. Buschmann, «Review on Bruun/Lörcher/Schömann/Clauwaert: The European Social Charter and the Employment Relation », AuR, 2017, 3, p. 27.

130 E. Shilton, «Public Rights and Private Remedies: Reflections on Enforcing Employment Standards through Grievance Arbitration », CLELJ, 2017, vol. 20, 1, p. 201.

131 I.C. Maggio, "La conciliazione e l'arbitrato nel diritto del lavoro: lo stato dell'arte », DRI, 2017, 1, p. 98.

132 Voir S. Banerjee, Z. Mahmood, op. cit.

133 P. Rataj, « Izbrane dobre prakse vrhovnih sodišč držav clanic EU in možnosti njihove uporabe v delovnih in socialnih sporih », E\&E, 2017, 2-3, p. 299. 


\section{B - NÉGOCIATION COLLECTIVE}

Le paysage des accords collectifs de travail est hétérogène et se présente sous différentes formes dans les États. Certains auteurs ont débattu des différentes structures de la négociation collective. Par exemple, ils distinguent la négociation à divers niveaux ${ }^{134}$, dans des structures centralisées ou décentralisées ${ }^{135}$, et le passage des conventions collectives interprofessionnelles aux conventions collectives sectorielles ${ }^{136}$. De nouvelles structures économiques ont en effet conduit à l'émergence de nouveaux niveaux de négociation, à l'instar de celui du groupe dont les résultats ont été évalués de manière ambivalente ${ }^{137}$. Des questions de frontières ont été soulevées entre les accords collectifs de travail et la loi ${ }^{138}$. Des problèmes spécifiques sont apparus en ce qui concerne la négociation collective et les accords collectifs de fonctionnaires ${ }^{139}$; problèmes auxquels nous serons confrontés de nouveau et de manière plus aiguë pour ce qui est du droit de grève des fonctionnaires. Tous les pays n'ont pas adopté de lois sur la conclusion et la mise en œuvre des accords collectifs de travail. Toutefois, même les négociations et les accords informels devraient bénéficier de la protection de la liberté d'association ${ }^{140}$. Enfin ${ }^{141}$, Liu a décrit la relation spéciale entre l'État, les syndicats et la négociation collective [en Chine], se référant au célèbre titre du film de Sergio Leone Le Bon, la Brute et le Truand.

II n'est donc pas surprenant que les directives juridiques impactent considérablement le contenu des accords collectifs de travail. Il semblerait qu'une flexibilisation du temps de travail, motivée par des considérations économiques et politiques se traduise à terme par un besoin accru de réglementation par négociation collective au moyen d'accords de négociation collective ${ }^{142}$. Les mêmes effets se sont produits avec la réglementation du travail temporaire. Compte tenu des risques particuliers liés au travail intérimaire, qui risque de détruire dans de nombreux pays les structures établies de représentation collective, la politique de négociation collective pourrait dès lors avoir une fonction corrective ${ }^{143}$. Dans certains pays, la loi, qui peut être altérée à tout moment par les accords collectifs de travail, s'est ainsi révélée très problématique. Il est légitime de se demander s'il incombe aux syndicats de revoir à la baisse les normes sociales. Cependant, en période d'affaiblissement syndical, ceux-ci sont susceptibles d'accepter certaines concessions, ce qui aurait été inenvisageable à d'autres époques. À cet égard, les notions de caractère raisonnable et de principe de proportionnalité pourraient utilement servir d'outils pour limiter le pouvoir excessif - de l'employeur - potentiellement exercé par le biais des conventions

134 M. Milan, Y. Ferkane, « Faut-il désormais craindre la négociation de groupe ?», RDT, 2017, 2, p. 76.

135 V. Bavaro, «Sulla prassi e le tendenze delle relazioni industriali decentrate in Italia (a proposito di un'indagine territoriale) », DRI, 2017, 1, p. 13.

136 Voir E. Carrizosa Prieto, op. cit.

137 M. Milan, Y. Ferkane, op. cit.

138 M. Magnani, «II rapporto tra legge e autonomia collettiva », DRI, 2017, 1, p. 1; M. Lai, op. cit.

139 M.L. Pérez Guerrero, "Los medios de solución extrajudicial de conflictos laborales en el sector público : problemática », TL, 2017, 140, p. 399.

140 E. González Biedma, « La negociación collectiva informal»,TL, 2017, 140, p. 121.

141 M. Liu, S. Kuruvilla, «The State, The Unions, and Collective Bargaining in China: The Good, the Bad and the Ugly ", CLLPJ, 2017, vol. 38, 2, p. 187.

142 Voir De La Flor Fernández, op. cit.

143 F. Vila Tierno, « Modalidades de contratación y empleo», TL, 2017, 140, p. 199. 
collectives $^{144}$. Les clauses dites "orphelin ", c'est-à-dire les clauses de disparité salariale basées uniquement sur les dates d'embauche différentes des personnes ${ }^{145}$, soulèvent également des difficultés. Tiraboschi ${ }^{146}$, pour sa part, reconnait les lacunes de la législation et de la politique de négociation collective en matière de "travail agile ». Enfin, Klein ${ }^{147}$ décrit la protection des Sozialkassenverfahren dans l'industrie du bâtiment; une institution allemande spécifique, qui sur la base de conventions collectives généralement déclarées contraignantes, garantit aux salariés de l'industrie du bâtiment la validité de leur droit aux congés et un régime de retraite d'entreprise. Ce système a récemment été entériné par des arrêts déterminants de la CEDH et de la Cour fédérale allemande du travail. La notion juridique de « liberté d'association négative » (si elle existe, elle est encore contestée) ne s'y oppose pas non plus.

\section{C - DROIT DE GRÈVE}

Le droit de grève est aujourd'hui menacé dans le monde entier. Cette problématique fait l'objet d'âpres discussions, au sein des organisations internationales comme au niveau national. De nombreux auteurs ont donc examiné les interventions des législateurs et des tribunaux nationaux en matière de droit de grève. D'autres se sont davantage penchés sur les lacunes des pays dans la mise en œuvre des normes internationales ou européennes, ou des exigences relatives aux garanties nationales en matière de liberté d'association. Enfin, de nombreux auteurs ont traité de questions particulières relatives à l'exercice du droit de grève.

En Allemagne, la loi sur le travail intérimaire a été légèrement réformée. Ces dernières années, l'embauche de travailleurs temporaires comme briseurs de grève est devenue une arme efficace utilisée par les employeurs pour enrayer l'action syndicale. Si cette méthode a certes été restreinte, il n'en demeure pas moins que des lacunes subsistent ${ }^{148}$. Les auteurs espagnols, en particulier, ont exprimé leurs préoccupations face à l'augmentation des interventions législatives sur le droit de grève. Lopez Lopez ${ }^{149}$ témoigne d'une dangereuse tendance à affaiblir le pouvoir de négociation des syndicats, par exemple en criminalisant les piquets de grève, sur la base de la réforme du marché du travail adoptée en Espagne en 2012. Suite à un arrêt de la Cour constitutionnelle espagnole, Pérez Rey ${ }^{150}$ a considéré qu'il s'agissait d'annihiler la grève technologique, tandis que Sánchez ${ }^{151}$ a mis l'accent sur

144 R. Santagata De Castro, «Indisponibilità del tipo, ragionevolezza e autonomia collettiva. Sul nuovo articolo 2, comma 2, decreto legislativo n. 81/2015», DRI, 2017, 2, p. 397.

145 Voir D. Gagne, MJ. Dupuis, op. cit.

$146 \mathrm{M}$. Tiraboschi, «I lavoro agile tra legge e contrattazione collettiva: la tortuosa via italiana verso la modernizzazione del diritto del lavoro », DRI, 2017, 4, p. 921.

147 T. Klein, «Die Sicherung der Sozialkassenverfahren im Baugewerbe », AuR, 2017, 2, p. 48.

148 T. Klein, D. Leist, « Kein Einsatz von Leiharbeitnehmern als Streikbrecher - Die Neuregelung in $\S 11$ Abs. 5 AÜG n.F. im Hinblick auf Auslegung, Schutzlücken, Rechtsfolgen und Durchsetzung », AuR, 2017, 3, p. 100.

149 J. Lopez, "Diminishing Unions' Agency: Weakening Collective Bargaining and Criminalizing Picketing in the Spanish Case », CLLPJ, 2017, vol. 38, 2, p. 169.

150 J. Pérez Rey, «El Tribunal Constitucional ante el esquirolaje tecnológico (o que la huelga no impida ver el fútbol)», RDS, 2017, 77, p. 151.

151 R. Sánchez lago, «Acción sindical y conflicto de derechos », DRL, 2017, 4, p. 361. 
la liberté d'expression dans le cadre des actions syndicales, également à la suite d'un arrêt de la Cour suprême.

Les garanties supranationales du droit de grève sont surtout le fait du droit de I'Organisation internationale du travail (OIT), du Pacte international relatif aux droits économiques, sociaux et culturels, de la Charte sociale européenne (révisée) et de la Convention européenne des droits de l'homme (CEDH). Ce «système à niveaux multiples » comprenant divers répertoires des droits de l'homme a gagné en portée juridique depuis que la Cour européenne des droits de l'homme, dans l'arrêt fondamental de la Grande Chambre Demir et Baykara sur l'article 11 de la CEDH, a adopté une approche globale, affirmant désormais prendre en compte les décisions des autres organes chargés de l'interprétation des normes dans les organisations internationales.

La Convention et la jurisprudence de la Cour européenne des droits de l'homme ont une force contraignante beaucoup plus importante que les autres instruments de droit international ou que la jurisprudence des organes chargés de leur interprétation. Cela pourrait éventuellement compenser le «traumatisme » causé par les arrêts Viking et Laval de la CJCE $^{152}$. Ce conflit s'intensifie pour ce qui est des entreprises publiques, telles que celles des transports ferroviaire et aérien, ainsi que plus globalement dans le secteur public, en particulier s'agissant des grèves menées par des fonctionnaires. Certains pays européens, à l'instar de l'Allemagne, n'ont pas encore pleinement intégré la jurisprudence claire de la Cour européenne des droits de l'homme selon laquelle les restrictions au droit de grève ne sont autorisées que dans quelques domaines de l'administration publique. Les arguments avancés contre le droit de grève dans ces domaines sont souvent fondés sur les principes de «services essentiels » ou d'« intérêt général », et mettent en avant l'exigence spécifique de loyauté de la part des fonctionnaires. Une autre occasion de débat s'est présentée lors de la conférence Zur Fundierung des Streikrechts im ILO-Normensystem (Fondements du droit de grève dans le système juridique de l'OIT), qui s'est tenue à Berlin les $1^{\text {er }}$ et 2 avril 2016, avec la participation de spécialistes du monde entier dont les interventions ont ensuite été publiées dans les revues de l'IALLJ.

Dans CLLPJ153 comme dans I'AuR ${ }^{154}$, Novitz a analysé la jurisprudence de I'OIT sur la grève dans le secteur public et les services essentiels. Du point de vue du droit international, les grèves dans les services essentiels ont également fait l'objet d'un article de Schlachter ${ }^{155}$ pour l'Allemagne et de Baylos Grau ${ }^{156}$ pour Castilla-La Mancha. Dans toutes ces analyses, la jurisprudence de la Commission d'experts de I'OIT (CEACR) sur les services essentiels a joué un rôle central. Mais le droit de mener des actions syndicales et l'avenir du mécanisme de surveillance de I'OIT'157, ainsi que les développements internationaux concernant la

152 J.P. Marguénaud, J. Mouly, C. Nivard, « Que faut-il attendre de la Cour européenne des droits de I'homme en matière de droits sociaux ?», RDT, 2017, 1, p. 12.

153 T. Novitz, "The Restricted Freedom to Strike: "Far-Reaching" ILO Jurisprudence on the Public Sector and Essential Services », CLLPJ, 2017, vol. 38, 3, p. 353.

154 T. Novitz, «Beamtenstreikrecht, Streik in der Daseinsvorsorge und das Recht auf politischen Streik - Teil 1 »,AuR, 8/9, p. 324; - Teil 2, AuR, 10, p. 376.

155 M. Schlachter, «Streiks in der Daseinsvorsorge aus völkerrechtlicher Sicht », AuR, 2017, 1, p. 10.

156 A. Baylos Grau, «Sindicato y crisis: conexiones y correspondencias », DRL, 2017, 2, p. 119.

157 J.M. Servais, "The Right to Take Industrial Action and the ILO Supervisory Mechanism Future ", CLLPJ, 2017, vol. 38, 3, p. 375. 
mise en œuvre du droit de grève ${ }^{158}$, ont été d'autres sujets tout aussi importants. Derrière ces conflits naissants se cache la querelle, qui a éclaté depuis plusieurs années au sein de I'OIT, au sujet de la garantie du droit de grève en vertu de la Convention 87 de I'OIT. Conformément à l'art. 37 de la Constitution de I'OIT, la Cour internationale de justice de La Haye devrait apporter une précision à ce sujet, même si elle n'a pas encore été saisie ${ }^{159}$.

Les grèves dans l'aviation civile et dans les aéroports, en particulier en Allemagne, ont donné lieu à quelques décisions de justice qui ont fait l'objet de quelques critiques par des académiques et des praticiens. Cela s'explique par le fait que des services publics, auparavant gérés de manière uniforme, ont été privatisés et décentralisés. II en résulte de nouvelles formes d'organisations qui génèrent de nouveaux conflits : les grèves de pilotes, du personnel de cabine ou des contrôleurs du transport aérien. Ce contexte est à l'origine de nouvelles questions très débattues : l'indemnisation des dommages causés par des grèves à des entreprises tierces ${ }^{160}$, la responsabilité syndicale pour des dommages au coût très élevé ${ }^{161}$, le droit procédural relatif à la protection juridique provisoire de l'action syndicale ${ }^{162}$, le contrôle par le juge des objectifs réels d'une grève et la théorie des œufs brouillés ${ }^{163}$. Selon cette théorie, un œuf pourri vient gâcher tous les autres œufs. Cette parabole étonnante, simpliste mais en même temps très explicite, a servi de base à la doctrine selon laquelle une revendication syndicale, jugée illégale par un tribunal, viendrait rendre illégal un conflit de travail dans sa totalité, et ce en dépit même du fait que le syndicat a porté plusieurs revendications parfaitement légitimes. C'est du moins l'avis de la Cour fédérale allemande du travail, qui s'oppose à l'arrêt de la Cour européenne des droits de l'homme n³6701/09, Hrvatski Liječnički Sindikat (HLS) v. Croatia. Dans cette affaire, la Cour européenne avait en effet confirmé qu'un conflit de travail dans lequel le syndicat avait présenté une demande ordinaire d'augmentation de salaire, en plus de deux revendications juridiquement problématiques, était légal.

158 N. Smit, "International Developments Regarding the Implementation of the Right to Strike ", CLLPJ, 2017, vol. 38, 3, p. 395.

159 C. La Hovary, "Article 37 of the ILO Constitution: An Unattainable Solution to the Issue of Interpretation? », CLLPJ, 2017, vol. 38, 3, p. 337.

160 U. Wendeling-Schröder, "Schadensersatz drittbetroffener Unternehmen bei Streiks ? ", AuR, 2017, 3, p. 96.

161 W. Däubler, « Haftung der Gewerkschaft für Millionenschäden ? », AuR, 2017, 6, p. 232.

162 R. Bram, "Aktuelle prozessrechtliche Fragen im einstweiligen Rechtsschutz von Arbeitskampfmaßnahmen », AuR, 2017, 6, p. 242.

163 A. Bücker, «Richterliche Erkenntnisse über wahre Streikziele und die Rühreitheorie », AuR, 2017, 8/9, p. 328. 